American Journal of Pharmacology and Toxicology 3 (1): 93-99, 2008

ISSN 1557-4962

(C) 2008 Science Publications

\title{
Cancer may be caused by the Immune Reaction
}

\author{
Richmond T. Prehn \\ Department of Pathology, University of Washington School of Medicine, \\ 5433 South Hudson St., Seattle, WA 98118, USA
}

\begin{abstract}
Hormesis-like effects of immunity have been documented during tumor-growth experiments in mice, quantitatively large immune-reactions have inhibited while lesser quantities of the same specific immune-reactants have been stimulatory to the growth of implanted tumors. Likewise, carcinogenesis has been inhibited by a high quantities of immune reactants, but positively stimulated by lower levels of the same reactants. One observation suggests that malignant transformation may seldom, if ever, occur in vivo if there is no immune reaction against the incipient neoplasm. These and many similar observations suggest a real danger that some vaccines might stimulate rather than inhibit tumor growth. These hormesis-like effects may help to provide explanations for a number of otherwise perplexing observations such as the following: (1): The fact that immunodepression for heart or kidney allografting results in an increased incidence of some tumors, especially leukemias and skin tumors, while others, such as those of breast and rectum, show a decreased incidence in these immunodepressed patients, (2): The possible dependency of carcinogenesis on an immune reaction, (3): The fact that adult Down's syndrome patients are unexpectedly resistant to solid tumors, especially breast-carcinoma, but have a very high incidence of leukemia, (4): The fact that Kaposi's sarcoma in an AIDS-patient often tends to flare as the patient's disease is ameliorated, (5): The sneaking through phenomenon, (6): Lastly, some peculiarities in the metastatic patterns of tumors of various types.
\end{abstract}

Key words: Carcinogenesis, tumor-immunostimulation, tumor-immunosurveillance, down-syndrome, kaposi's-sarcoma

\section{INTRODUCTION}

Many toxins exhibit a nonlinear dosage effect called hormesis. This study is limited to a consideration of the hormetic-like effects of tumor-specific immunity on carcinogenesis and tumor growth, hormetic effects of hormones or of other agents on tumor growth are not discussed.

The fact that an immune reaction may, under some circumstances, act to enhance rather than inhibit neoplastic growth has been known for many years ${ }^{[1]}$. The first convincing demonstration of a biphasic doseresponse curve in a totally syngeneic system was probably a study with 3-methylcholanthrene (MCA)induced mouse sarcomas ${ }^{[2]}$. A fixed number of immunogenic sarcoma cells was mixed with varied numbers of immune spleen cells, i.e., spleen cells from mice that had previously grown the same tumor. The resulting tumor-growths, when such an admixture was implanted subcutaneously in radiated and thymectomized hosts, were compared with controls consisting of that same tumor mixed with like numbers of nonimmune spleen-cells or with no spleen-cells. It was found that relatively small numbers of the admixed

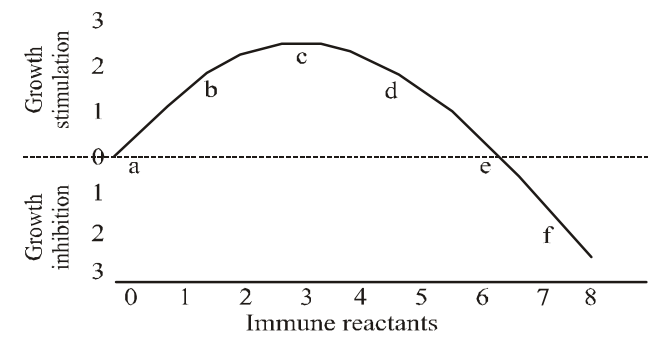

Fig. 1: Idealized curve derived from data in ${ }^{[2]}$. Numbers and lettered points have been added arbitrarily to aid the discussion

immune-cells stimulated tumor-growth while larger numbers were inhibitory. The biphasic dose-response curve, shown in Fig. 1, is an idealized depiction of these data ${ }^{[2]}$. The fact that the host animals had been radiated and thymectomized suggested that a blockage of an inhibitory immunity by a host-generated blocking antibody was an unlikely explanation for the apparently stimulated tumor-growth.

Although the above experiment demonstrated a biphasic curve for the titration of the immune reaction against tumor implants, would an hormetic curve also 
be found for the effect of various quantities of the immune reaction on carcinogenesis? Mice were radiated and thymectomized in order to drastically reduce their immune capacities ${ }^{[3]}$. The immune capacities were then restored to varying degrees by the intraperitoneal injection of varied numbers of normal, adult, syngeneic spleen-cells. Carcinogenesis was then induced by the subcutaneous administration of wafers containing a uniform dosage of MCA. Three groups were thus created: no immunologic restoration, minimal restoration and maximal restoration. The number of tumors/number of animals in each group at approximately 100 days after implantation of the wafers were as follows: no restoration $=48 / 100(48 \%)$, low restoration $=68 / 94(72 \%)$, high restoration $=38 / 97$ $(39 \%)$. The minimal restoration group was distinguished from the other two by $\mathrm{p}<0.001$. Thus, it is apparent that carcinogenesis occurred most efficiently in association with a positive, but minimal, immune reaction, a result in accordance with the tumor-implant data $^{[2]}$.

In subsequent years, a large literature has appeared suggesting that growth stimulation by a tumor-specific immune reaction may be exhibited in a variety of tumor systems $^{[4]}$ and by a variety of supposedly cytotoxic immune reactants. In addition to unsegregated spleencells, separated immune-elements such as antiserum ${ }^{[5,6]}$, macrophages ${ }^{[7,8]}$, lymphotoxin ${ }^{[9]}$, or various lymphoid cell populations ${ }^{[10,11]}$, acting either separately or in algebraic aggregate, have stimulated tumor growth. Although the biphasic hormetic dose-response curve has been rigorously demonstrated only for unfractionated immune spleen cells and for antibody with compliment, at this time it seems possible that any entity capable of reacting with an antigen on a neoplastic cell might exhibit the same hormetic-like phenomenon, i.e., stimulation of the target cells at low titer, but tumor-inhibition or tumor-killing at high. The mechanisms that may underlie the biphasic responsecurve have not been determined, but have been the subject of some speculation ${ }^{[12,13]}$. The phenomenon is clearly dependent, at least in part, upon the quantity rather than the species of the reactive immune elements. However, there is little doubt that some immune elements are more stimulatory than are others. Macrophages seem to be particularly likely to stimulate tumor growth, especially growth of breast carcinomas, but they are sometimes inhibitory, at least in the dosages examined and particularly in nasopharingeal and prostate carcinomas ${ }^{[14-21]}$. A recent report claims that an influx of macrophages turns the angiogenic switch $^{[20]}$.

\section{TUMORIGENESIS IN HUMAN ALLOGRAFT RECIPIENTS}

An apparently increased incidence of cancers in patients immunodepressed to facilitate either kidney or heart allo-transplantation was one of the first evidences supporting the immunosurveillance hypothesis ${ }^{[22]}$. However, it soon became apparent that the tumor incidences in such patients were not uniformly increased. Although some tumors, such as lymphoreticular cancers and cancers of the skin were markedly more prevalent than they were among normal control populations ${ }^{[22]}$, some carcinomas such as those of the breast ${ }^{[23]}$ and of the rectum ${ }^{[24]}$ showed a reduced incidence in the immunodepressed patients. How are such data to be interpreted?

Animal experimentation, largely in mice, has shown that tumors vary widely in their immunogenicities, in general, sporadic, spontaneous tumors in mice have little detectable immunogenicity while carcinogen-induced tumors or those induced by a variety of oncogenic viruses tend to be more highly immunogenic ${ }^{[25]}$. Among tumors of apparently identical histologic type, produced in one and the same animal by the same dosage of the same chemical carcinogen, both antigenic specificity and degree of immunogenicity may vary widely ${ }^{[26]}$. Consequently, each tumor elicits a different dosage of immune reactants, each tumor necessarily falls at a different place along the immune response curve (Fig. 1). Human breast and rectal cancers, because immunodepression lessens their incidence, presumably fall near c, or to the left of c, while leukemias and skin cancers, whose incidence is increased in immunodepressed subjects, will usually fall far to the right of point c (Fig. 1). It is apparent that the increased incidence of these tumors may be caused by either decreased inhibition or increased stimulation of tumor growth depending upon whether the reaction in the immunodepressed patients falls to the right or to the left of point e (Fig. 1). Thus, the anomalous behavior of different tumor-types among human allograft recipients is neatly explained by the hormetic-like effect of varied dosages of immune reactants, while other explanations are not excluded, this interpretation, based upon an hormetic effect, has considerable heuristic appeal.

\section{IMMUNE DEPENDENCY}

Lappé and Prehn studied the development of mouse skin papillomas in response to initiation with 3methylcholanthrene (MCA $)^{[27]}$. Papillomas were produced by treating the skin of a normal mouse with a 
sub-carcinogenic dosage of MCA and then grafting that skin onto a syngeneic mouse whose immunologic capacity had been raised or lowered by various techniques. This technique presumably by-passed the immunodepressive effects of the carcinogen. The trauma of transplantation served as a promoter of the initiated skin. Analysis of the effect of a moderate immunodepression of the host mice, in the Lappé/Prehn papilloma study, showed that the moderate immunodepression increased papilloma-incidence, increased the incidence of malignancy and delayed papilloma-regression ${ }^{[27]}$. The result is consistent with either increased immunostimulation or decreased immunoinhibition of the papillomas depending upon whether the reaction's location is actually to the left or to the right respectfully of point e on the immunereaction-curve (IRC-Fig. 1). In either case, the reaction would presumably have been well to the right of $\mathrm{c}$ and analogous to the increased skin-cancer incidence in the immunodepressed allograft patients. A contrary result was obtained if the host mice had been immunostimulated rather than immunodepressed. Almost identical results were reported in analogous experiments by Outzen ${ }^{[28]}$.

Subsequently, the skin-papilloma system was reinvestigated with a slightly modified protocol ${ }^{[29]}$. Andrews methodology was to transplant skin, previously exposed to MCA, to an allogeneic rather than to a syngeneic host. In order to permit the grafts to survive in the allogeneic host, the host animals were maximally immunodepressed by thymectomy, $\mathrm{x}-$ radiation and weekly injections of antithymocyte serum. No evidence of surviving immune-function could be found, so the reaction was presumably close to a on the IRC (Fig. 1). Nonetheless, about $80 \%$ of the induced papillomas regressed, but, most surprisingly, none progressed to malignancy. Although the trauma of transplantation was adequate to promote the formation of papillomas, a significant immune reaction was apparently necessary, in this system, for significant malignant-transformation, but not for regression of the trauma-promoted papillomas ${ }^{[29]}$.

Further data demonstrating the relative tumorenhancing action of low-dose immunity, relative to the effect of either lower or higher dosages, was found in the relationship between the latencies of tumors and their immunogenicities. Among 154 sarcomas induced in mice by a constant dosage of MCA, it was observed that the tumors of shortest latencies had intermediate levels of immunogenicity ${ }^{[30]}$. Thus, the first 34 tumors to arise (latency of 8-12 wks) had an average immunogenicity (growth advantage of tumor implants in control versus growth in immunized recipients) of
2.84 , the next 38 (latency of 13-15wks) had an increased average immunogenicity of 4.48 , while tumors with only1.5 to non detectable levels of immunogenicity had an average latency in excess of 19 wks. Thus, the tumors of intermediate immunogenicity grew the fastest. These differences were highly significant and ancillary data suggested that they were indeed caused by differences in the strengths of the immune reactions ${ }^{[30]}$. One would have expected, according to the surveillance hypothesis, that tumors of the least average immunogenicity would have had the shortest, rather than the longest, average latency. (These were average results, some tumors grew rapidly despite low immunogenicities).

These, as well as many other observations ${ }^{[31,32]}$, challenge the widely held immune-surveillance hypothesis. A developing immune-response to a tumor probably must, regardless of the ultimate immunogenicity of the tumor, be weak before it grows strong. In view of the tumor-stimulatory effect at the weak end of the immune-response curve, there seems to be little possibility of surveillance, at least as the surveillance hypothesis was originally conceived, i.e., elimination of most tumors virtually at their incipiency by an immune reaction. Apparently, for immunity to have an inhibitory effect upon the development of a cancer, the tumor's immunogenicity, as in most human skin carcinomas, must be sufficiently high so that later inhibition of the tumor's growth by an immune reaction will more than counterbalance earlier immunostimulation. For further discussions of the surveillance hypothesis ${ }^{[4]}$.

The fact that the dose-response curve is biphasic may explain why numerous studies in mice of the effects of immunodepression have shown little effect, thus leading some investigators to conclude, probably erroneously, that neither immune surveillance nor immune stimulation are important influences on carcinogenesis ${ }^{[12,33]}$. It is apparent that if immunodepression moves the system from near $d$ to near b (Fig. 1) neither tumor inhibition nor tumor stimulation will be seen. The fact that Stutman ${ }^{[33]}$ found immunodepression to have so little effect on carcinogenesis, suggests that in actuality most induced tumors in normal mice probably fall somewhere near $d$ on the curve, but are moved to near $\mathrm{b}$ by moderate immunodepression. However, in some cases still greater immunodepression might cause the system to move further to the left, beyond $b$, thus resulting in poorer tumor growth, in contrast, even lesser degrees of immunodepression might cause relatively enhanced growth by moving the reaction further to the left, but short of point $b$. The net result might well be nil. The 
biphasic shape of the curve makes it imperative that a very wide range of points on the curve be studied before drawing conclusions.

A possible consequence of the hormetic curve: namely, that tumors may have antigens primarily because an immune response is favorable and perhaps even necessary for initiating tumor growth in vivo. It seems that, during progression, a tumor may tend to adjust both its immunogenicity and its susceptibility to the resulting immunity to some level, greater than null, that is more nearly optimum for the growth of that particular tumor ${ }^{[34,35]}$.

\section{TUMORS AMONG DOWN SYNDROME PATIENTS}

In recent decades, patients with Down syndrome have become longer lived, thus it is now possible to compare the tumor incidence among Down patients with that in a comparably aged normal-population. The result is a quite unexpected tumor-profile. The incidences of most solid tumors, but especially those of the female breast, are extremely low among Down patients ${ }^{[36]}$. On the other hand, leukemias are much more prevalent than they are in normal subjects ${ }^{[36]}$.

A characteristic of Down patients is a susceptibility to infection ${ }^{[36]}$ and specific immune deficits have been described $^{[37]}$. Although the Down tumor-profile ${ }^{[36]}$ differs from that of the immunodepressed heart or kidney allograft patients, a similar explanation of the unexpected Down-profile, based primarily on the hormetic-like quality of the immune-reaction curve, may still be possible.

The Down patient's immune-reaction to a tumor obviously differs in at least two crucial ways from that of the immunodepressed allograft-recipient: in the magnitude as well as in the duration of the immune deficit. While the magnitudes of the immune-system deficits are not readily ascertained, it is clear that in a Down patient, in possible contrast to the allograft patient, the immune reaction is relatively depressed throughout a tumor's entire course. Thus, the immune reaction in the Down patient is abnormally low during the early phase of tumor formation, when the tumor is probably dependent upon immunity ${ }^{[35]}$. By contrast, among the allograft patients, many of the tumors may have been initiated prior to the start of the immunodepression and therefore would not have experienced the lack of early immunostimulation. Many of the tumors seen in allografted patients probably started long before the immunodepression as is dramatically suggested by the observation of Halpern who showed that men retain the same constant probability of dying from lung cancer for the next 10 to 20 years after they stop smoking ${ }^{[38]}$. Analogous results have been found in human breast cancer ${ }^{[13]}$. Thus the long latency of many human tumors may help to explain the fact that, although mammary cancer is reduced in both down and allograft groups, the reduction is more spectacular among the Down patients $^{[36]}$. However, this is just one among many possibilities.

Both allografted and Down patients show an excess of leukemias, in both cases the best explanation might be that there may be a compensatory hyperplasia of one or more elements of the immune mechanism itself.

\section{KAPOSI'S SARCOMA IN HIV INFECTED PATIENTS}

Another observation, consistent with the hormetic nature of the immune response curve, has been seen in connection with the behavior of Kaposi's sarcoma. This tumor is a common feature of the acquired immunodeficiency syndrome (AIDS), but it often flares as recovery from immunosuppression occurs during effective AIDS treatment ${ }^{[39]}$. Furthermore, those AIDS patients who present with Kaposi's live longer than those who lack the tumor at the time of presentation $^{[40,41]}$ A reasonable interpretation is that this tumor grows best when the immune reaction of the host is less than normal, but not too low. As with any clinical observation, to an even greater degree than in mouse work, any interpretation is merely the best bet among many known and unknown possible confounders.

\section{THE SNEAKING THROUGH PHENOMENON}

The dosage of an implanted tumor often has a profound effect upon its growth. Usually, if an immunogenic tumor-implant fails to grow in syngeneic recipients, increasing the dose of tumor will overcome the resistance. Sometimes drastically reducing the dosage will also result in growth of a recalcitrant implant, hence the appellation sneaking through. The phenomenon has sometimes been observed even in previously immunized mice ${ }^{[42,43]}$.

While the mechanism of the phenomenon has not to my knowledge been established, I cannot resist suggesting that it may be related to the hormesis effect as diagrammed on the IRC curve (Fig. 1). Let us postulate that a tiny dose of tumor stimulates the animal's immune mechanism only weakly and that the tumor is thereby stimulated to grow, perhaps it is near c on the IRC. Thus, the tumor sneaks through. A larger 
tumor-dose might produce a larger reaction and move the tumor further to the right on the IRC, perhaps past e where inhibition might prevent tumor growth. A yet larger dose of tumor might overwhelm the immune system and temporarily soak up enough of the immune reactants to actually reduce the effective size of the reaction, in that event, the placement on the IRC might again be moved, at least temporarily, to the left, perhaps past e where the tumor would again be stimulated to grow.

\section{THE NEWBORN TUMOR-HOST}

Can the hormetic character of the immune response curve explain the anomalous nature of tumor growth in the newborn? It is well known that the immune capacity of a mouse at birth is virtually nonexistent, but a number of papers have reported that most syngeneic tumors, as well as embryonic tissues, grow poorly when implanted in these immunologically immature-mice as compared with their growths in adult recipients ${ }^{[4,45]}$. The relative inhibition of growth is gradually attenuated as the recipients grow older and is largely dissipated in mice by the 6th day after birth. These observations appear to fit well with the previously discussed dependency of a new tumor on an immune response, a response which, in this case, is largely absent until after the 6th day. That the immune response of the young is very different from that in the adult is supported by direct histologic examination of human tumors ${ }^{[46]}$.

The fact that normal fetal tissues, as well as tumors, grow poorly in the newborn is another similarity between malignancies and embryonic tissues and the poor growth of both in the newborn might depend in part upon the lack of an adequate immune reaction to the carcinoembryonic antigens which are shared by both tumors and fetal tissues ${ }^{[47]}$.

\section{CONCLUSIONS}

The immune reaction demonstrates, in its action upon carcinogenesis and tumor growth, the phenomenon of hormesis. The nonlinear nature of the immune reaction-curve needs to be taken into account in devising immunological cancer-preventions and therapies. The possibility of doing actual harm by inducing or administering therapeutically inadequate quantities of immune reactants may be very real.

\section{REFERENCES}

1. Kaliss, N., 1965. Immunological enhancement and inhibition of tumor growth: Relationship to various immunological mechanisms. Federation Proc., 24: 1024-1029.
2. Prehn, R.T., 1972. The immune reaction as a stimulator of tumor growth. Science, 176: 170-171.

3. Prehn, R.T., 1972. Immunostimulation of chemical oncogenesis in the mouse. Int. J. Cancer, 20: 918-922.

4. Prehn, R.T., 2006. An adaptive immune reaction may be necessary for cancer development. Theor. Biol. Med. Model, 3: 6.

5. Shearer, W.T., G.W. Philpott and C.W. Parker, 1973. Stimulation of cells by antibody. Science, 182: 1357-1359.

6. Heidrick, M.L., W.L. Ryan and G.L. Curtis, 1978. Stimulation of malignant skin cells by antibody to normal skin cells of mice. J. Natl. Cancer Inst., 60: 1419-1425.

7. Evans, R., 1978. Macrophage requirement for growth of a murine fibrosarcoma. Br. J. Cancer, 37: 1086-1089.

8. Mantovani, A., 1978. Effects on in vitro growth of murine macrophages isolated from sarcoma lines differing in immunogenicity and metastasizing capacity. Int. J. Cancer, 22: 741-746.

9. Hofsli, E., R. Austgulen and J. Nissen-Meyer, 1987. Lymphotoxin-induced growth stimulation of diploid human fibroblasts in the presence and absence of gamma interferon. Scand. J. Immunol., 26: 585-588.

10. Norbury, K.C., 1977. In vitro stimulation and inhibition of tumor cell growth mediated by different lymphoid cell populations. Cancer Res., 37: 1408-1415.

11. Tsuda, T., Y.T. Kim, G.W.W. Siskind, A. DeBlasio and R. Schwab, 1987. Role of thymus and T-cells in slow growth of B16 melanoma in old mice. Cancer Res., 47: 3097-3100.

12. Outzen, H.C., 1980. Development of carcinogeninduced skin tumors in mice with varied states of immune capacity. Int. J. Cancer, 26: 87-92.

13. Prehn, R.T., 2007. Does the immune reaction cause malignant transformation by disrupting cell-to-cell or cell-to-matrix communications? Theor. Med. Biol. Model, 4: 16.

14. Peng, J., T. Dind, L.M. Zheng and J.Y. Shao, 2006. Influence of tumor-associated macrophages on progression and prognosis of nasopharyngeal carcinoma. Ai Zheng., 25: 1340-1345.

15. Luo, Y., H. Zhou, J. Krueger, C. Kaplan, S.H. Lee, C. Dolman, D. Markowitz, W. Wu, C. Liu, R.A. Reisfeld and R. Xiang, 2006. Targeting tumor-associated macrophages as a novel strategy against breast cancer. J. Clin. Invest., 116: 2132-2141. 
16. Shimura, S., G. Yang, S. Ebara and T.M. Wheeler, 2000. Reduced infiltration of tumor-associated macrophages in human prostate cancer: Association with cancer progression. Cancer Res., 60: 5857-5861.

17. Pollard, J.W., 2004. Tumour-educated macrophages promote tumour progression and metastasis. Natl. Rev. Cancer, 4: 71-78.

18. Davey, K., S.N. Yi, W.J. Hawthorne, A.M. Lehnert, H. Ha, J.K.W. Wong, N. van Rooijen, P.J. O'Connell, A.T. Patel and S.N. Walters, 2003. T cell activated macrophages are capable of both recognition and rejection of pancreatic islet xenografts. J. Immunol., 170: 2750-2758.

19. Bingle, L., N.J. Brown and C.E. Lewis, 2002. The role of tumour associated macrophages in tumour progression: implications for new anticancer therapies. J. Pathol., 196: 254-265.

20. Lin, E.Y., J.F. Li, L. Gnatovskiy, Y. Deng, L. Zhu, D.A. Grzesik, H. Qian, X.N. Xue and J.W. Pollard, 2006. Macrophages regulate the angiogenic switch in a mouse model of breast cancer. Cancer Res., 66: 11238-11246.

21. Tsutsui, S., K. Yasuda, K. Suzuki, K. Tahara, H. Higashi and S. Era, 2005. Macrophage infiltration and its prognostic implications in breast cancer: the relationship with VEGF expression and microvessel density. Oncol. Rep., 14: 425-431.

22. Penn, I., 1988. Tumors of the immunocompromised patient. Annu. Rev. Med., 39: 63-73.

23. Stewart, T.H.M. and G.H. Heppner, 1997. Immunological enhancement of breast cancer. Parasitol., 115: S141-S153.

24. Stewart, T.H.M., 1997. Reduced incidence of rectal cancer, compared to gastric and colonic cancer, in a population of 73, 076 men and women chronically immunosuppressed. Clin, Cancer Res., 3: 51-55.

25. Prehn, R.T., 1976. Tumor progression annd homeostasis. Adv. Cancer Res., 23: 203-236.

26. Basombrio, M.A. and R.T. Prehn, 1972. Antigenic diversity of tumors chemically induced within the progeny of a single cell. Int. J. Cancer, 10: 1-8.

27. Lappé, M.A. and R.T. Prehn, 1969. Immunologic surveillance at the macroscopic level: nonselective elimination of premalignant skin papillomas. Cancer Res., 29: 2374-2387.

28. Outzen, H.C., 1980. Development of carcinogeninduced skin tumors in mice with varied states of immune capacity. Int. J. Cancer, 26: 87-92.
29. Andrews, E.J., 1971. Evidence of the nonimmune regression of chemically induced papillomas in mouse skin. J. Natl. Cancer Inst., 47: 653-665.

30. Prehn, R.T. and G.L. Bartlett, 1987. Surveillance, latency and the two levels of MCA-induced tumor immunogenicity. Int. J. Cancer, 39: 106-110.

31. Prehn, R.T. and L.M. Prehn, The autoimmune nature of cancer. 1987. Cancer Res., 47: 927-932.

32. Prehn, R.T. and L.M. Prehn, 1989. The flip side of tumor immunity. Arch. Surg., 124: 102-106.

33. Stutman, O., 1975. Immunodepression and malignancy. Adv. Cancer Res., 22: 261-422.

34. Prehn, R.T., 1970. Analysis of antigenic heterogeneity within individual 3methylcholanthrene-induced mouse sarcomas. J. Natl. Cancer Inst., 45: 1039-1045.

35. Prehn, R.T., 1977. Immunostimulation of the lymphodependent phase of neoplastic growth. J. Natl. Cancer Inst., 59: 1043-1049.

36. Bérnard, J., N. Béron-Gaillard and D. Satgé, 2005. Down's syndrome protects against breast cancer: is a constitutional microenvironment the key? Int J. Cancer, 113: 168-170.

37. Franceschi, C., F. Licastro, M. Chiricolo, F. Bonetti, M. Zannotti, N. Fabris, E. Mocchegiani, M.P. Fantini, P. Paolucci and M. Masi, 1981. Deficiency of autologous mixed lymphocyte reactions and serum thymic factor level in Down's syndrome. J. Immunol., 126: 2161-2164.

38. Halpern, M.T., 1993. Patterns of absolute risk of lung cancer mortality in former smokers. J. Natl. Cancer, 85: 457-464.

39. Leidner, R.S. and D.M. Aboulafia, 2005. Recrudescent Kaposi's sarcoma after initiation of HAART: a manifestation of immune reconstitution syndrome. AIDS Patient Care STDS, 19: 635-644.

40. Moss, A.R., G. McCallum, P.A. Volberding and P. Bacchetti, 1984. Mortality associated with mode of presentation in immune deficiency syndrome. $\mathbf{J}$. Natl. Cancer Inst., 73: 1281-1284.

41. Rivin, B.E., J.M. Monroe, B.P. Hubschman and P.A. Thomas, 1984. AIDS outcome: a follow-up. N. Engl. J. Med., 211: 857.

42. Marchant, J., 1969. Sarcoma induction in mice by methylcholanthrene (Antigenicity tests of sarcomas in thymus grafted and control animals). Br. J. Cancer, 23: 383-390.

43. de Boer, R.J. and P. Hogeweg, 1986. Interactions between macrophages and T-lymphocytes: Tumor sneaking through intrinsic to helper cell dynamics. J. Theor. Biol., 120: 331-351. 
44. Tumyan, B.G., S.N. Zinzar, G.J. Svet-Moldavsky and B.I. Leitina,, 1974. Suppression of tumors and transplants of fetal digestive tract growing in newborn mice. J. Natl. Cancer Inst., 52: 63-67.

45. Svet-Moldavsky, G.J., S.N. Zinzar and N.Y. Karmanova, 1976. Inhibition of tumor and fetal tissue growth in newborn recipients. Ann. N.Y. Acad. Sci., 276: 328-342.
46. Vakkila, J., R. Jaffe, M. Michelow, M.T. Lotze., 2006. Pediatric cancers are infiltrated by macrophages and contain a paucity of dendritic cells: a major nosologic difference with adult tumors. Clin. Cancer Res., 12: 2049-2054.

47. Hammarstrom, S., 1999. The carcinoembryonic antigen (CEA) family: Structures, suggested functions and expression in normal and malignant tissues. Semin. Cancer Biol., 9: 67-81. 